\title{
Integer Encoding Genetic Algorithm for Optimizing Redundancy Allocation of Series-parallel Systems
}

\author{
Xueli Cheng ${ }^{1, *}$, Linchao $\mathrm{An}^{1}$ and Zhenhua Zhang ${ }^{2}$ \\ ${ }^{1}$ Department of Mechanical Engineering, Henan Institute of Technology, Xinxiang 453002, China \\ ${ }^{2}$ School of Mechanical and Civil Engineering, Curtin University, Perth 6102, Australia
}

Received 13 January 2019; Accepted 2 March 2019

\begin{abstract}
Reliability redundancy allocation is a combinatorial optimization problem, and numerous intelligent evolutionary algorithms (e.g., genetic algorithm and ant colony optimization) have been proposed to solve it. However, various shortcomings, such as problem specificity and high complexity, hinder their applications. An integer encoding genetic algorithm, namely, integer matrix chromosome encoding scheme, was proposed to improve the effectiveness and computational efficiency of redundancy allocation for series-parallel systems and represent the component mixing in subsystems with integers. The related crossover with a binary window and mutation using a matrix with random float numbers was developed to perform combinatorial evolution. The adjusting operator was designed to guarantee the feasibility of chromosomes, combined with the non-dominated sorting genetic algorithm (NSGA-II) in which a constraint Pareto dominance was introduced to handle design constraints without external coefficients. Numerical and engineering examples of an agricultural Internet of Things for greenhouse planting were provided to illustrate the effectiveness of the proposed algorithm. Results show that the proposed novel algorithm can solve a typical model for reliability redundancy allocation, i.e., a non-maintained bi-state series-parallel system with active redundancy and component mixing strategy. The constraint Pareto dominance is introduced on the basis of the traditional NSGA-II to avoid the complexity and instability of penalty function approaches. The constructed three-objective redundancy allocation problem model can measure the trade-off relationship among three objectives, namely, system reliability, cost, and weight. The improved NSGA-II has the best stability when the optimized value for crossover probability is 0.98 and the mutation probability is set to a small value. Advantages of the presented model and method include its convenience and suitability for different genetic evolutionary platforms.
\end{abstract}

Keywords: Reliability redundancy allocation, Series-parallel system, Combinatorial optimization, Integer encoding genetic algorithm, NSGA-II

\section{Introduction}

With the increasing sophistication of today's engineering systems, considerable concerns are being placed on system reliability; thus, redundancy allocation becomes an important aspect in system reliability design. Reliability redundancy allocation appoints the components' redundancy strategies for subsystems to optimize the reliability of the entire system under the constraints of system cost and/or weight. On the basis of different system redundancy strategies and constraints, the redundancy allocation problem (RAP) for series-parallel systems may consider the following options [1]: (a) active or cold-standby redundancy, (b) component non-mixing or mixing schemes, (c) nonmaintained or maintained systems, and (d) bi- state or multistate systems. Reliability redundancy allocation is crucial in various types of mechanical and electrical systems.

Generally, RAP is evaluated by formulating nonlinear integer optimization problems, which consider a single objective (e.g., maximizing system reliability). The singleobjective RAP is known as an NP-hard problem [2], which

*E-mail address: chengxueli2005@126.com

ISSN: $1791-2377$ @ 2019 Eastern Macedonia and Thrace Institute of Technology. All rights reserved. doi:10.25103/jestr.121.15 has been solved using a number of optimization approaches for different formulations, as summarized by Wang [3]. Ardakan [4] presented a problem formulation and solution methodology for the general single-objective RAP when the redundancy strategy of all subsystems is a cold standby. Teimouri [5] proposed two meta-heuristic methods to solve single-objective RAPs. Considering the conflicting objectives among system reliability, cost, and weight, multiobjective RAP (MORAP) was recently solved with evolutionary algorithms. The encoding scheme of individuals is an important aspect for solving RAPs with evolutionary algorithms. As an integer combination problem, RAP differs from the traditional design optimization problems of discrete or continuous variables in a predefined range. Hence, the encoding scheme of chromosomes and related evolutionary operators become the key to redundancy allocation, which brings a considerable challenge to researchers.

Numerous studies have been conducted on the MORAP evolutionary algorithm. Evolutionary algorithms include the genetic algorithm with dynamic penalty functions [6], ant colony optimization (ACO) [7], and penalty-guided bee search algorithms [8], in which specific chromosome encoding schemes and evolutionary operators must be developed. Unstable factors may be introduced with different penalty coefficient values. Moreover, comparisons 
among the three objectives, namely, maximized system reliability, max-min approach, and minimized standard deviation, are inadequate to guide the selection of the most appropriate reliability optimization objective for solving specific RAPs. Therefore, establishing a multi-objective optimization RAP model and developing an integer encoding genetic algorithm that optimizes the redundant allocation for series-parallel systems are urgent problems to be solved.

On this basis, the present study explores an integer coding genetic algorithm to optimize the redundant allocation for series-parallel systems and develops the related integer crossover, mutation, and adjusting operators. Comparisons among three reliability objectives are also considered. This study aims to solve RAP for series-parallel systems accurately, thereby providing a reference for the redundant allocation of various types of electromechanical systems.

\section{State of the art}

At present, numerous studies have been conducted on multiobjective algorithms for the redundant allocation of seriesparallel systems. Chambari [9] presented a bi-objective redundancy allocation model for maximizing reliability and minimizing cost, and the model was solved using nondominated sorting genetic algorithm II (NSGA-II) and multiobjective particle swarm optimization. However, the multiple objectives lacked contrast. Yeh [10] presented a simplified swarm optimization for solving multi-level seriesparallel RAPs, but did not involve component mixing. Zio [11] proposed a multi-objective evolutionary algorithm for the RAP of multi-state systems to acquire the Pareto fronts with three objectives. However, the algorithm was unsuitable for parallel systems. Liang [12] developed a multi-objective variable neighborhood search algorithm for solving RAPs. However, the multiple objectives lacked contrast. Yeh [13] introduced an orthogonal simplified swarm optimization scheme that combined repetitive orthogonal array testing, population re-initialization, and simplified swarm optimization for solving the RAP of serialparallel systems with mixing components. This scheme was computationally efficient but did not involve active redundancy. Feizollahi [14] studied RAP in series-parallel systems with a cold standby strategy. The linear mixedinteger programming and binary-equivalent models for the cold standby RAPs were proposed for the first time. However, they were only applicable to non-repairable systems. Ardakan [15] introduced a new redundancy strategy, namely, mixing redundancy, in multi-objective optimization RAPs, which could improve the reliability of any system with redundant components. However, this strategy was unsuitable for non-maintenance systems. To solve the bi-objective reliability RAP for series-parallel systems, Garg [16] developed a fuzzy model, which was converted into a crisp model by using the expected values of fuzzy numbers and considering the preference of the decision maker regarding cost and reliability objectives. Finally, the obtained crisp optimization problem was solved with particle swarm optimization. Their results were compared with those of genetic algorithms. However, the multiple objectives lacked contrast. Yeh [17] proposed a collaborative parallel simplified swarm algorithm to solve the RAP for smart sensor systems in the Internet of Things (IoT). This scheme could solve the RAP of serial-parallel systems with mixing components, but it did not involve active redundancy. Tavakkoli-Moghaddam [18] proposed a genetic algorithm for the RAP of series-parallel systems when the redundancy strategy could be selected for individual subsystems. However, this algorithm did not involve multi-objective optimization. To determine the optimal combination of the number of components for each subsystem that maximizes the system reliability under total fuzzy cost and weight constraints, Mousavi [19] proposed an improved fruit fly optimization algorithm to solve the RAP for series-parallel systems. However, the multiple objectives lacked contrast. Coit [6] proposed a single-objective redundancy allocation approach for the series-parallel system via genetic algorithm to fit the RAP with active redundancy and a bi-state system. Chambari [9] introduced a transformation mechanism to solve RAP with two objectives, in which the integers in a chromosome were mapped to float numbers in $(0,1)$ scale to suit the genetic algorithms. However, the premise was the none-mixing scheme for RAP. Integer encoding scheme and ACO [7] were proposed to solve MORAP. However, ACO evolutionary operators might be developed specifically, leading to the high complexity of the algorithm. Maciej [20] developed a modified selection operator combined with classical NSGAII and removed the crowded operator from NSGA-II. Combinations of multiple selection modifiers were investigated and the best configurations were identified. However, various modifications could result in the convergence, expansion, or uniformity of achieved Pareto fronts. He [21] analyzed a mixed redundancy strategy with instant switching for series-parallel systems and established the redundancy allocation model to minimize redundancy configuration costs under the transient availability and job completion rate constraints. However, the multiple objectives lacked contrast. Wang [22] applied the universal generating function method to calculate the system availability and combined the particle swarm and local search algorithms to solve the RAP for series-parallel systems. However, the multi-objective model was not established.

In the aforementioned studies, specific chromosome encoding schemes and evolutionary operators may be developed, and a few unstable factors may be introduced with different penalty coefficient values. Moreover, comparisons among three objectives, namely, maximized system reliability, max-min approach, and minimized standard deviation, are inadequate to guide the selection of the most appropriate reliability optimization objective for solving specific RAP. In this study, the RAP was encoded with an integer matrix, in which the related integer crossover, mutation, and adjusting operators were developed. The integer encoding redundancy allocation model was solved with traditional NSGA-II, in which constraint Pareto dominance was introduced to avoid the complexity and instability in the penalty function approaches. The effectiveness of the algorithm was demonstrated on RAPs with one, two, and three objectives, and its robustness was proven with sensitivity analysis. Contributions of the method are as follows: the integer matrix encoding scheme, related integer crossover, mutation, and adjusting operators of chromosomes are simple to use, and the model can be transplanted conveniently in other multi-objective genetic algorithms to solve the MORAP for serial-parallel systems easily. Numerical and engineering examples of an agricultural IoT for greenhouse planting were provided to illustrate the effectiveness of the proposed algorithm. 
The remainder of this study is organized as follows: Section 3 describes the RAP, which is followed by the chromosome encoding scheme and system performance formulations. The RAP optimization model is constructed, and the key evolutionary operators and computational framework of the algorithm is discussed. Section 4 presents the numerical examples and comparisons for single- and multiple-objective optimizations. Section 5 presents an engineering example of an agricultural IoT for greenhouse planting. Section 6 draws the conclusions.

\section{Methodology}

\subsection{Mathematical model}

For a non-maintained bi-state series-parallel system with active redundancy and component mixing scheme, as shown in Fig. 1, each subsystem has $m_{j}$ available components $(j=$ $1,2, \ldots, s$, and $s$ is the number of series subsystems), and different types of components can be paralleled in one subsystem. Supposing that the maximum number of parallel components in each subsystem is $\mathrm{n}$ and that the minimum number of components in subsystems is $n_{\min }$, the reliability allocation for acquiring the maximum system reliability under system cost and weight constraints is an NP-hard problem [23].

The major assumptions or limitations of the mathematical model for non-maintained systems are as follows:

- The component states and related system only have two options, namely, working and failure.

- The component attributes (i.e., reliability, cost, and weight) are known and deterministic.

- Only the active redundancy strategy is considered.

- No component repair or preventive maintenance is available.

- Failures of the components are independent events.

- Components within the same subsystem can be different.

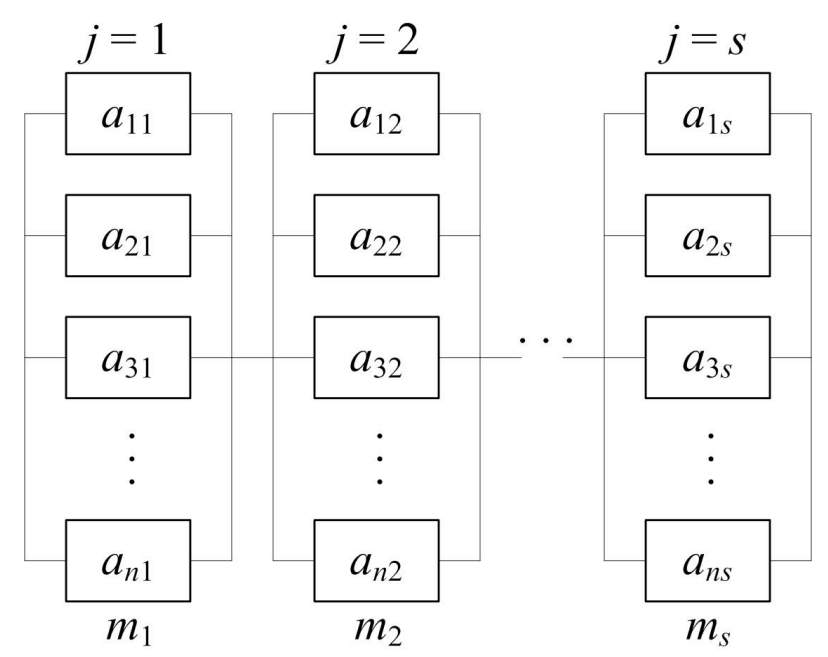

Fig. 1. Series-parallel system

An integer matrix encoding with $\mathrm{n}$ rows and s columns is proposed. The available components in each subsystem are coded as $1,2, \cdots, m_{j}$, where $m_{j}$ represents the number of available components in the $j_{t h}$ subsystem. The $n$ elements in column $j$ are random integers in $0,1,2, \cdots, m_{j}$, in which
0 denotes that a component is not used in the position, and an integer in 1 to $m_{j}$ exhibits that an available component is used in the position. Fig. 2 illustrates an encoding example of RAP with 14 subsystems, in which the maximum number of parallel components in each subsystem is $n=4$. For example, subsystem 1 is parallel to components 1,3 , and 4 . The encoding scheme introduces 0 to represent the empty redundancy position.

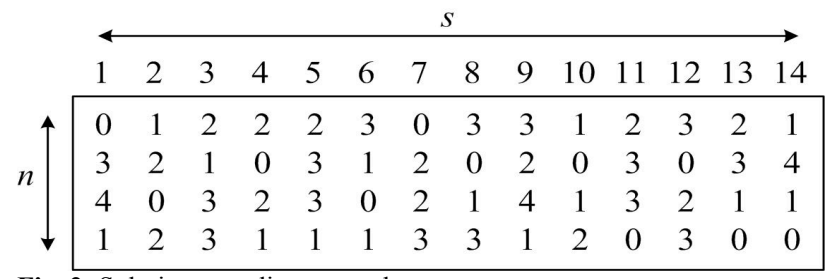

Fig. 2. Solution encoding example

Let a chromosome for RAP be represented with $A_{n \times s}$, and elements in the matrix are $a_{i, j}(i=1,2, \ldots, n ; j=1$, $2, \ldots, s)$. The mathematical model for RAP is described as follows:

The number of active redundancy components $\left(x_{j}\right)$ used in subsystem $j$ is as follows:

$x_{j}=\sum_{i=1, a_{i, j} \neq 0}^{n} a_{i, j} / a_{i, j}$

where $a_{i, j}$ is an integer in the $i$ th row and $j$ th column in $A_{n \times s}$ to represent a component used in subsystem $j$ or an empty position without components and $x_{j} \leq n$.

The reliability for one subsystem is determined by

$R_{j}=1-\prod_{i=1, a_{i, j} \neq 0}^{n}\left(1-r_{a_{i, j}, j}\right)$

where $r_{a_{i, j}, j}$ is the reliability of component $a_{i, j}$ in the $j$ th subsystem.

The reliability for the entire system is as follows:

$R_{s}=\Pi_{j=1}^{s} R_{j}$

where $R_{j}$ is the reliability for the $j$ th subsystem and $R_{s}$ indicates the system reliability.

The cost of the entire system is as follows:

$C_{s}=\sum_{j=1}^{s} C_{j}=\sum_{j=1}^{s} \sum_{i=1, a_{i, j} \neq 0}^{n} c_{a_{i, j}, j}$

where $c_{a_{i, j}, j}$ is the cost of component $a_{i, j}$ in the $j$ th subsystem, $C_{j}$ represents the cost of the $j$ th subsystem, and $C_{s}$ denotes the system cost.

The weight of the entire system is expressed as

$W_{s}=\sum_{j=1}^{s} W_{j}=\sum_{j=1}^{s} \sum_{i=1, a_{i, j} \neq 0}^{n} w_{a_{i, j}, j}$ 
where $w_{a_{i, j}, j}$ indicates the weight of component $a_{i, j}$ in subsystem $j, W_{j}$ refers to the weight of the $j$ th subsystem, and $W_{s}$ is the system weight.

A RAP optimization model with three objectives can be illustrated as

Maxmize $R_{s}$, Minimize $C_{s}$, and Minimize $W_{s}$

Subject to: $C_{s} \leq C_{\max }$

$$
\begin{aligned}
& W_{s} \leq W_{\max } \\
& n \geq n_{\text {min }}
\end{aligned}
$$

where $C_{\max }$ and $W_{\max }$ are the maximum allowed cost and weight of the system, respectively. $n_{\min }$ denotes the minimum number of components used in each subsystem, such as $n_{\min }=1$.

RAP optimization also aims to maximize the minimum reliability in subsystems and minimize the standard deviation of reliabilities in subsystems as follows:

$$
\text { Maximize (Minimum } R_{j}, j=1,2, \mathrm{~L}, s \text { ) }
$$

Minimize $\left(\operatorname{Std} \operatorname{Dev} R_{j}, j=1,2, \mathrm{~L}, s\right)$

where $R_{j}$ is the reliability of the $j$ th subsystem. Minimum $R_{j}$ represents the minimum reliability in all subsystems, and Std Dev refers to the standard deviation of reliabilities in all subsystems. Each parameter can be used to replace the maximized system reliability objective to acquire further balanced reliability values in the subsystems.

\subsection{Multi-objective genetic algorithm for RAP}

The genetic operators for RAP differ from the simulated binary crossover and polynomial mutation [24] for the multiple float variables in traditional genetic algorithms.
Thus, special crossover and mutation operators should be developed to evolve the integers in each column with the available components in each subsystem. Given the populations' random initialization, crossover, and mutation in genetic algorithms, the adjusting operator should be used prior to performance evaluation to guarantee the feasibility of the chromosomes for RAP. In this study, NSGA-II [7] is improved to solve the MORAP for series-parallel systems.

\subsubsection{Crossover}

The original tournament selection is used in the improved NSGA-II (INSGA-II) (NSGA-II with integer-matrix encoding) to form parents, and a crossover operator with a binary window is proposed. Let the two parent matrixes waiting to be crossed be $P 1_{n \times s}$ and $P 2_{n \times s}$. A binary matrix $B_{n \times s}$ with 0 or 1 element is randomly generated. For all elements in $B_{n \times s}$, if $b_{i, j}=0$, then elements in the $i$ th row and $j$ th column in child chromosomes $C 1_{n \times s}$ and $C 2_{n \times s}$ are equivalent to the corresponding elements in $P 1_{n \times s}$ and $P 2_{n \times s}$. If $b_{i, j}=1$, then elements in the $i$ th row and $j$ th column in $P 1_{n \times s}$ and $P 2_{n \times s}$ are exchanged and assigned to the corresponding positions in $C 1_{n \times s}$ and $C 2_{n \times s}$. A crossover probability $p_{c}$ is used. $p_{c}$ is generally set as a large value, e.g., $0.9 \leqslant p_{c} \leqslant 1.0$, to improve the global searching capability of the genetic algorithm. Fig. 3 shows a crossover example for integer-matrix encoding, in which the randomly generated binary window is $B_{4 \times 14}$. Given that $b_{2,1}=1$, elements $p 1_{2,1}=0$ and $p 2_{2.1}=3$ are exchanged in the child chromosomes to be $c 1_{2,1}=3$ and $c 2_{2,1}=0$, respectively. Other elements in the child chromosomes obey the same theory.

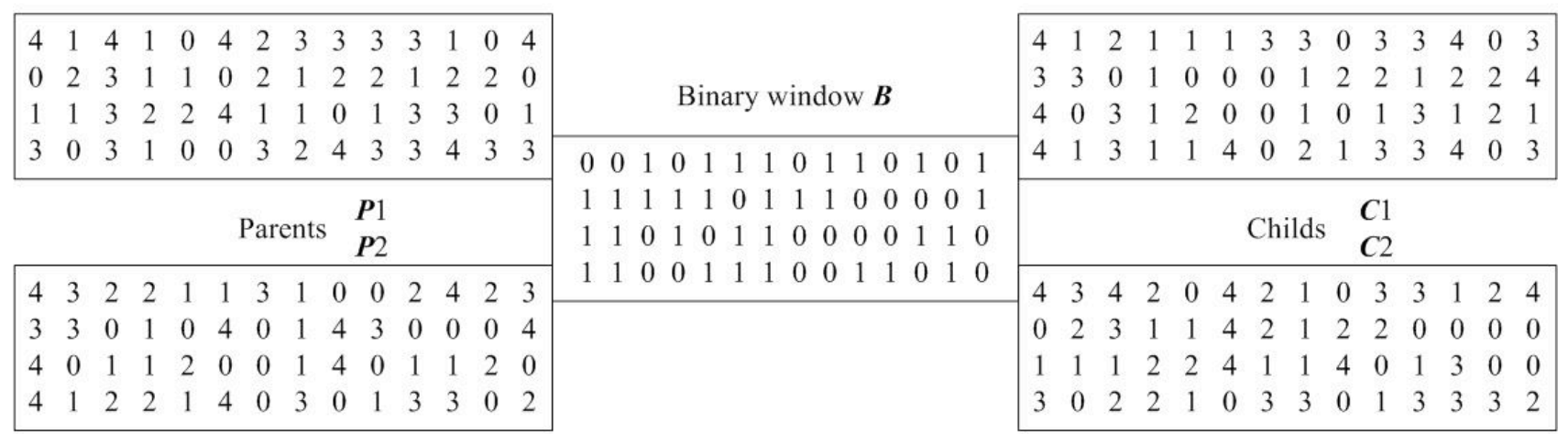

Fig. 3. Crossover example

\subsubsection{Mutation}

The mutation scheme for solving RAP is to mutate the chromosomes on the basis of a random float matrix. All chromosomes in the population should be mutated. For each chromosome $A_{n \times s}$, a matrix $M_{n \times s}$ is generated, in which each element in the matrix is a random float number in $(0,1)$ scale. The mutation probability pm is commonly set as a small value, such as $p_{m}=1 /$ (number of design variables).
When $m_{i, j}<p_{m}$, an integer between 0 and $m_{j}$ is randomly generated to replace the original $a_{i, j}$. Fig. 4 presents an example of mutation. Let $p_{m}=0.1$. When an element in the random matrix $m_{3,1}=0.08<p_{m}$, element $a_{3,1}$ is changed from 1 to 3 . 


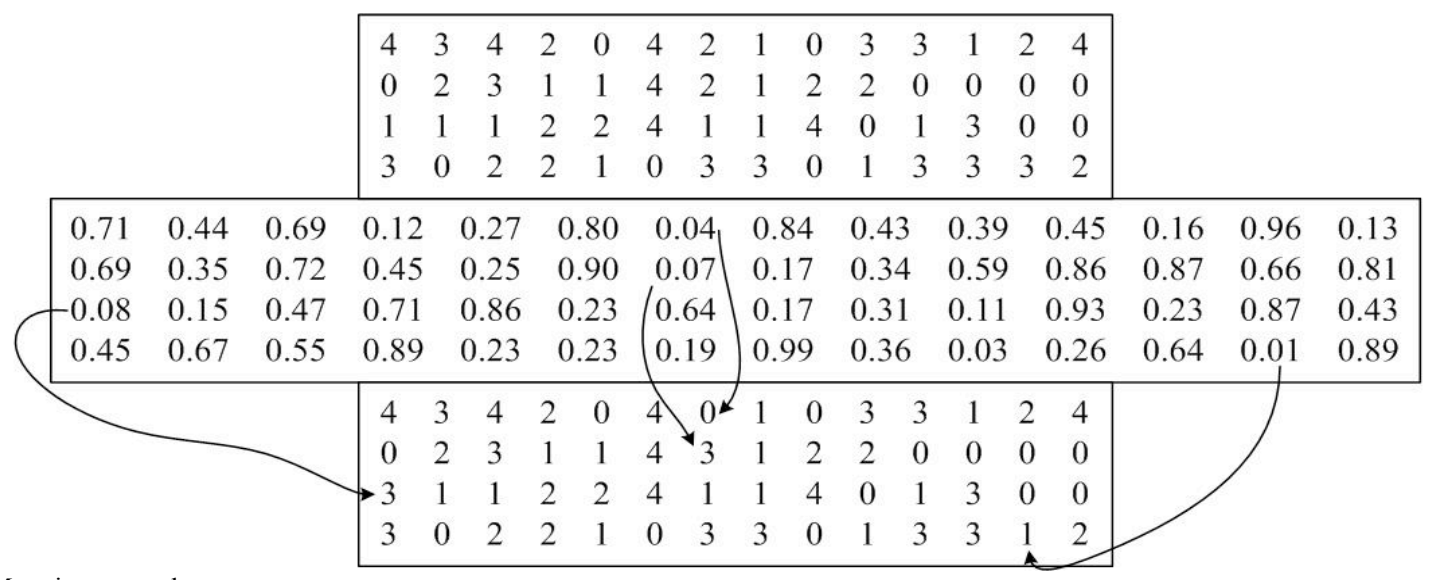

Fig. 4. Mutation example

\subsubsection{Guaranteeing the feasibility of individuals}

The minimum number of paralleled components in each subsystem is set as an integer $n_{\min }$, and $n_{\min } \geqslant 1$. Situations (e.g., columns with all zero elements or smaller number of parallel components in subsystems than $n_{\min }$ ) may be generated due to the populations' random initialization, crossover, and mutation in genetic algorithms. Thus, before the performance measure for each chromosome, the adjusting operator should be introduced to guarantee the feasibility of each chromosome for RAP. The adjusting operator for one column includes the following steps: First, the number of active redundancy components $x_{j}(j=1,2, \cdots$, s) in the subsystem is calculated. Second, if $x_{j}<n_{\min }$, then $n_{\min }-x_{j}$ zero elements will be selected randomly and replaced with random integers in 1 to $m_{j}$, where $m_{j}$ is the number of available components for subsystem $j$. If $x_{j} \geqslant$ $n_{\text {min }}$, then column $j$ is feasible, and each column in the chromosome should be checked.

\subsubsection{INSGA-II framework}

Fig. 5 illustrates the INSGA-II workflow, and the main steps are described as follows:

Step 1 The population with $p_{\text {num }}$ random chromosomes is initialized. Each chromosome is an integer matrix with size $n \times s$ and the iteration counter $t=1$.

Step 2 The feasibility of each chromosome or solution in the population is checked. The infeasible solutions are adjusted to be feasible in this step.

Step 3 The performance of each chromosome is evaluated. The crowding distance of each chromosome is calculated, and the chromosomes are ranked with constraint Pareto dominance.

Step 4 Tournament selections are performed to form a child population.

Step 5 Integer matrix crossovers with binary windows are performed for the child population.

Step 6 Mutations with probability pm are performed for the child population.

Step 7 Adjusting operators are executed to check and modify the chromosomes to be feasible in the child population.

Step 8 The performance of chromosomes in the child population is evaluated.

Step 9 The parent and child populations are merged, and the crowding distance of chromosomes is calculated. Individuals in the temporary population are ranked with constraint Pareto dominance, and a new parent population is generated. Step 10 Assessment is performed to determine if the maximum number of iterations $G_{\max }$ is satisfied. If $t=G_{\max }$, the non-dominated solutions in the parent population are outputted as Pareto optimal solutions; otherwise, $t=t+1$. Return to Step 4 to continue the iteration.

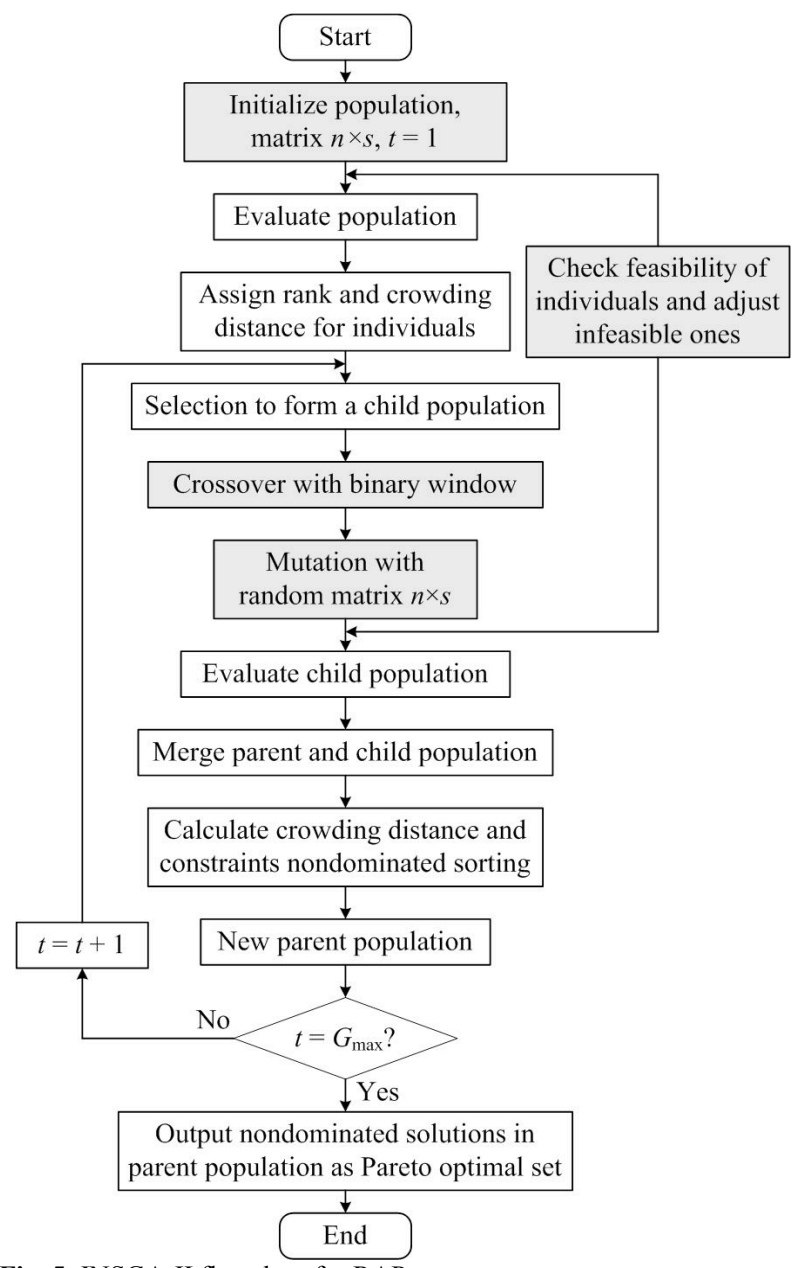

Fig. 5. INSGA-II flowchart for RAP

The main differences between INSGA-II and NSGA-II with float design variables are illustrated in boxes with grey backgrounds in Fig. 5, including the population initialization, binary window crossover, float matrix mutation, and chromosomes' feasibility adjusting operators. The adjusting operator is performed after the initialization and mutation of the population. 
The proposed chromosome encoding scheme, crossover, mutation, and adjusting operators can also be combined with other multi-objective genetic algorithms, such as strength Pareto evolutionary algorithm 2 (SPEA2) and Epsilon multiobjective evolutionary algorithm [25]. The RAP model with integer matrix encoding has satisfactory suitability for different genetic platforms.

\section{Result analysis and discussion}

A standard RAP test originally proposed by Fyffle et al. was used to demonstrate INSGA-II. The problem is a RAP for a non-maintained bi-state series-parallel system with active redundancy and mixing scheme. Coit and Smith [6] solved this problem with a genetic algorithm without restricting the component mixing, and the maximum redundancy level $n$ selected from available component types in the subsystem is 8.

This study assumes the minimum redundancy levels $n_{\text {min }}=2$ and $n=4$ for all subsystems. Input data from document [7] of the component characteristics for this problem are summarized. INSGA-II is developed on the basis of standard NSGA-II in the Kanpur Genetic Algorithms Laboratory in $\mathrm{C}$ language and run on a desktop computer with a dual $2.4 \mathrm{GHz}$ CPU and $2 \mathrm{~GB}$ RAM. The mathematical models with different objective numbers and evolutionary parameters are described as follows.

\subsection{Comparison of objectives for redundancy allocation} To the compare different optimization objectives for RAP, three single-objective models were set up: maximizing the system reliability (Max $R_{s}$ ), maximizing the minimum reliability in subsystems (Max-min), and minimizing the standard deviation of reliabilities in subsystems (Min Std Dev). The design constraints were $C \leqslant C_{\max }, W \leqslant W_{\max }, \mathrm{n}=4$, and $n_{\text {min }}=2$. Two cases were evaluated to compare the optimization results for RAP under different constraints, where $C_{\max }=130$. Through computational experiments, the evolutionary parameters of INSGA-II for the singleobjective RAP were population $p_{\text {num }}=300$, maximum iterations $G_{\max }=500$, crossover probability $p_{c}=0.98$, mutation probability $p_{m}=1 / s$, and $s=14$.

Table 1 presents the performances of RAP solutions acquired by INSGA-II with three different objectives in the two cases, in which $R_{s}$ is the system reliability, $R_{\text {sub-min }}$ denotes the minimum reliability in subsystems, and $D_{\text {sub }}$ refers to the standard deviation of reliabilities in all subsystems. The data in Table 1 are graphically shown in Fig. 6 in that:

(a) Max $R_{s}$ acquired the maximum system reliability in Fig. 6(a), and relatively good $R_{s u b-\min }$ and $D_{s u b}$ were achieved with Max $R_{s}$ in Figs. 6(b) and (c).

(b) The max-min approach acquired the maximum $R_{\text {sub-min }}$ as shown in Fig. 6(b), but caused the maximum $D_{\text {sub }}$ in Fig. 6(c) and the relatively low system reliability in Fig. 6(a).

(c)The Min Std Dev objective acquired the minimum Dsub in Fig. 6(c), but caused the lowest $R_{s}$ and $R_{s u b-m i n}$ for $W_{\max }=191$, as shown in Figs. 6(a) and (b).

The results in $W_{\max }=191$ had larger change rates than those in $W_{\max }=159$ because the RAP flexibility was improved with a large system weight to achieve enhanced solutions for redundancy allocation. Table 1 and Fig. 6 show that the Max $R_{s}$ objective achieved the maximum improvement in system reliability with relatively few sacrifices in the minimum and standard deviation of reliabilities in subsystems. These results prove that Max $R_{s}$ is the most suitable objective for RAP with INSGA-II.

Table 1. Performance comparison among different objectives

\begin{tabular}{l|l|l|l|l|l|l}
\hline \multirow{2}{*}{ Performance } & \multicolumn{2}{|l|}{ Case 1: $C_{\max }=130, W_{\max }=191$} & \multicolumn{2}{l}{ Case 2: $C_{\max }=130, W_{\max }=159$} \\
\cline { 2 - 6 } & Max $R_{s}$ & Max-Min & Min Std Dev & Max $R_{s}$ & Max-Min \\
\hline$R_{s}$ & 0.9852 & 0.9799 & 0.9710 & 0.9519 & 0.9322 \\
$R_{\text {sub-min }}$ & 0.9971 & 0.9975 & 0.9964 & 0.9900 & 0.9900 \\
$D_{\text {sub }}$ & $8.56 \mathrm{E}-04$ & $8.59 \mathrm{E}-04$ & $7.27 \mathrm{E}-04$ & $27 \mathrm{E}-04$ & $34 \mathrm{E}-04$ \\
\hline
\end{tabular}
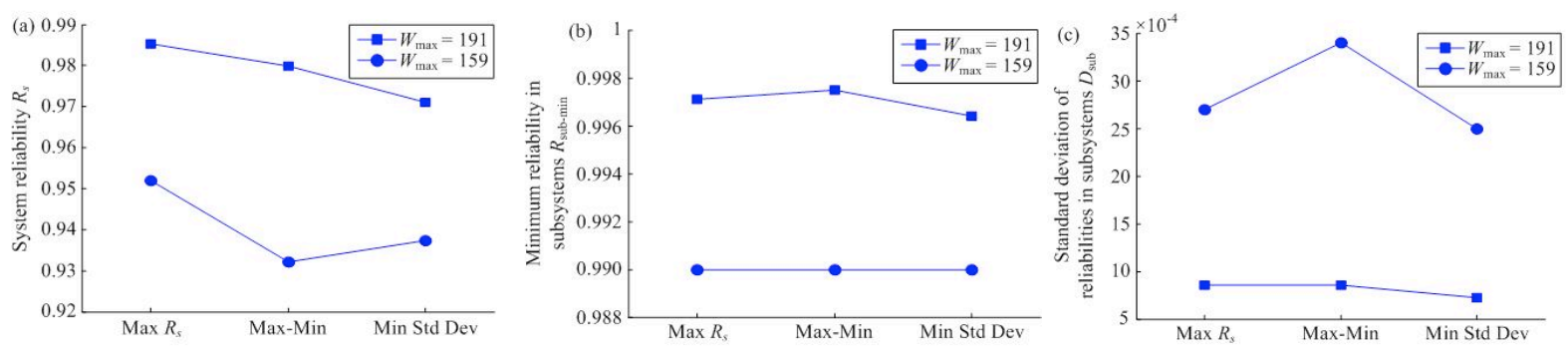

Fig. 6. Comparison of performances using different objectives, (a) system reliability, (b) minimum reliability in subsystems, and (c) standard deviation of reliabilities in subsystems

\subsection{Optimization for RAP with Single Objective}

With the maximizing system reliability objective, singleobjective models were applied to test the effectiveness of INSGA-II. By setting the maximum cost $C_{\max }=130$ and maximum weight from $W_{\max }=191$ to 159,33 singleobjective models were constructed with different design constraints. By using the same evolutionary parameters presented in Section 4.1, 10 runs were performed for each 
model because of the random initialization in INSGA-II. The maximum (Max $R_{s}$ ), minimum (Min $R_{s}$ ), average (Avg $R_{s}$ ), and standard deviation of the 10 reliabilities (Std Dev) for each model were shown. The maximum possible index (\%MPI) [7], [8] was introduced to compare the reliabilities with INSGA-II and GARAP [6]. Fig. 7 illustrates the results. (a) The main areas of the solid line in Fig. 7 were above $y=0$, which showed that the maximum reliabilities acquired by INSGA-II were larger than the correspondences with GARAP.

(b) A few regions in the dot chain line were smaller than $y=0$, which showed that a minimum reliability with INSGA-II was worse than GARAP in a small degree.

(c) The redundancy level $n=4$ for INSGA-II was smaller than that for GARAP in which $n=8$, and Max $R_{s}$ values in INSGA-II were better than those in GARAP. From these comparisons, INSGA-II achieved similar RAP results with GARAP by using small iteration numbers.

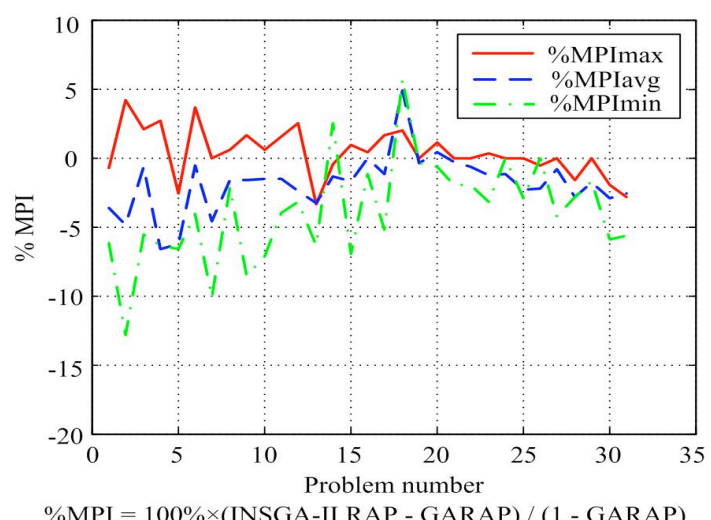

Fig. 7. Comparison of GARAP and INSGA-II RAPs

Fig. 8 shows the standard deviation comparisons for all models, in which the solid line with INSGA-II had smaller fluctuations than the dotted line with GARAP.

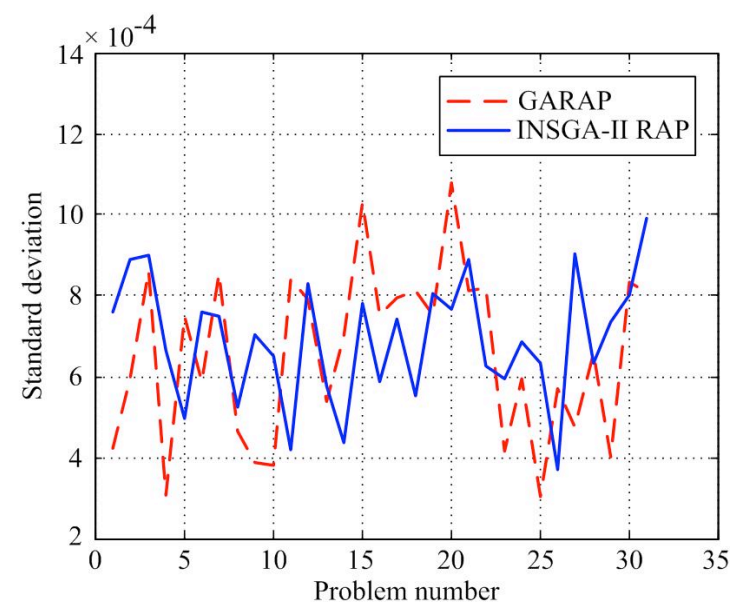

Fig. 8. Comparison of standard deviations between GARAP and INSGA-II RAPs

Therefore, INSGA-II had better computational stability than GARAP. The performance comparisons proved the effectiveness and stability of INSGA-II for RAP with a single objective.

\subsection{Optimization for RAP with Multiple Objectives}

A two-objective model for the standard RAP was constructed as follows:
Minimize $\left(-R_{s}\right)$, Minimize $C_{s}$;

Subject to: $C_{s} \leq 130$;

$$
W_{s} \leq 191
$$

Where: $n_{\min }=2$, and $n=4$

where the maximized reliability $R_{s}$ is multiplied with $(-1)$ to transform the model into a two-objective minimization problem.

Fig. 9 shows the acquired Pareto front with the INSGAII platform in Section 3.2.4 and the evolutionary parameters in Section 4.1. Fuzzy set theory [26] was introduced to select a compromised best solution. The solution was commonly the "knee point" [27] in Pareto optimal solutions. Moreover, fuzzy set theory was only effective for Pareto fronts with two minimization or maximization objectives, but it was unsuitable for the Pareto front with one maximized and minimized objective. Table 2 presents the redundancy allocation schemes and related performances for the points of maxima of reliability only, minima of cost only, compromise best, and baseline design in Fig. 9. Fig.9 and Table 2 show that: (a) the Pareto front with relatively good boundary and diversity characteristics for RAP with two objectives could be acquired by INSGA-II. (b) Neither maxima of reliability only $R_{s}=0.9846$ nor minima of cost only $C_{s}=87$ was superior to the baseline design in view of both objectives. (c) Compromised solutions $R_{s}=0.9727$ and $C_{s}=99$ acquired a higher reliability and lower cost than the baseline designs $R_{s}=0.9708$ and $C_{s}=120$, in which the optimization amounts were $0.19 \%$ and $17.5 \%$, respectively.

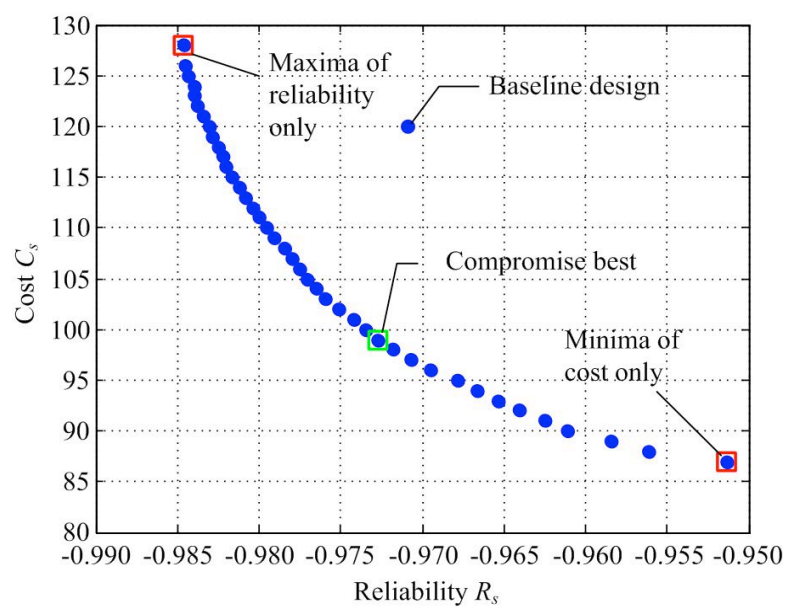

Fig. 9. Pareto fronts acquired by INSGA-II for RAP with two objectives

Thus, the compromised best solution for RAP with two objectives reduced the system cost by $17.5 \%$, improved the system reliability by $0.19 \%$, and increased the sacrifice on the system weight by $15.9 \%$. Moreover, the Pareto front had good boundary and diversity characteristics. These results prove the effectiveness of INSGA-II for RAP with two objectives. The system cost $C_{s}=99$ of the compromised best solution in Table 2 was small, the weight of the compromised best solution was $W_{s}=191$ to attain the upper bound in the weight constraint in Formula (9). Thus, the system cost and weight were conflicting objectives, and the system weight should be substantial to achieve a small cost and large system reliability. The multi-objective optimization for RAP could exhibit the relationship between design objectives and constraints better than the single- 
objective optimization. This result will help the solution trade-off with preferences for decision makers.

Table. 2. Results for redundancy allocation optimization with two objectives using INSGA-II

\begin{tabular}{c|c|c|c|c}
\hline $\begin{array}{c}\text { Descr } \\
\text { iption }\end{array}$ & $\begin{array}{c}\text { Baseline } \\
\text { design }\end{array}$ & $\begin{array}{c}\text { Compromise } \\
\text { d } \\
\text { Best }\end{array}$ & $\begin{array}{c}\text { Minima } \\
\text { of } \\
\text { cost only }\end{array}$ & $\begin{array}{c}\text { Maxima of } \\
\text { reliability only }\end{array}$ \\
\hline 1 & 3330 & 1110 & 2220 & 3330 \\
2 & 1100 & 1100 & 2200 & 1100 \\
3 & 4440 & 1340 & 1300 & 4440 \\
4 & 3330 & 1110 & 1110 & 1333 \\
5 & 2220 & 2220 & 1100 & 2220 \\
6 & 2200 & 3400 & 3300 & 2200 \\
7 & 1300 & 3300 & 2200 & 3300 \\
8 & 1130 & 1111 & 1110 & 1111 \\
9 & 3300 & 1100 & 1100 & 1200 \\
10 & 2220 & 2220 & 2220 & 3330 \\
11 & 1100 & 1100 & 1100 & 3300 \\
12 & 1111 & 1111 & 1110 & 1111 \\
13 & 2200 & 1100 & 1100 & 1200 \\
14 & 3300 & 1400 & 2300 & 3400 \\
$R_{s}$ & 0.9708 & 0.9727 & 0.9514 & 0.9846 \\
$C_{s}$ & 120 & 99 & 87 & 128 \\
$W_{s}$ & 170 & 191 & 186 & 190 \\
\hline & & & & \\
\hline
\end{tabular}

A three-objective RAP model with maximized system reliability, minimized cost, and minimized weight was solved by INSGA-II with the design constraints in Formula (9) and similar evolutionary parameters. The Pareto optimal solutions shown in Fig. 10 had relatively good boundary and diversity characteristics. As shown in Fig. 10, the system reliability was increased with the large system weight and cost. Considering the reliability and cost, the trend of Pareto optimal solutions was similar to that in Fig. 9. Given that fuzzy set theory [26] was only effective for fronts with two objectives, the "knee point" method [27] should be used in the design trade-off for Pareto fronts with three objectives. The compromised selection process and results from Fig. 10 were not presented in this paper.

\subsection{Sensitivity analysis}

The sensitivity analysis optimized different values of the key parameters in INSGA-II to analyze the stability of the algorithm and acquire the suitable ranges for the parameters. The key parameters in INSGA-II were the crossover probability pc and mutation probability $p_{m}$.

Fig. 11 illustrates the Pareto fronts acquired with different $p_{c}$ values with $p_{m}=1 / s \quad(s=14)$ by using the same two-objective model (Formula (9)), that is, population number $p_{\text {num }}$ and maximum iteration $G_{\max }$ in Section 4.3. The Pareto front with $p_{c}=0.90$ was dominated by the fronts with $p_{c}=0.98$ or $p_{c}=1.0$. The boundary characteristic of the front with $p_{c}=0.98$ was better than that with $p_{c}=1.0$. Thus, the stability of INSGA-II with different crossover probabilities was proven, and the optimized value for the crossover probability was 0.98 . These results show that sufficient crossover operators were necessary for an enhanced convergence of INSGA-II under the predefined number of iterations.

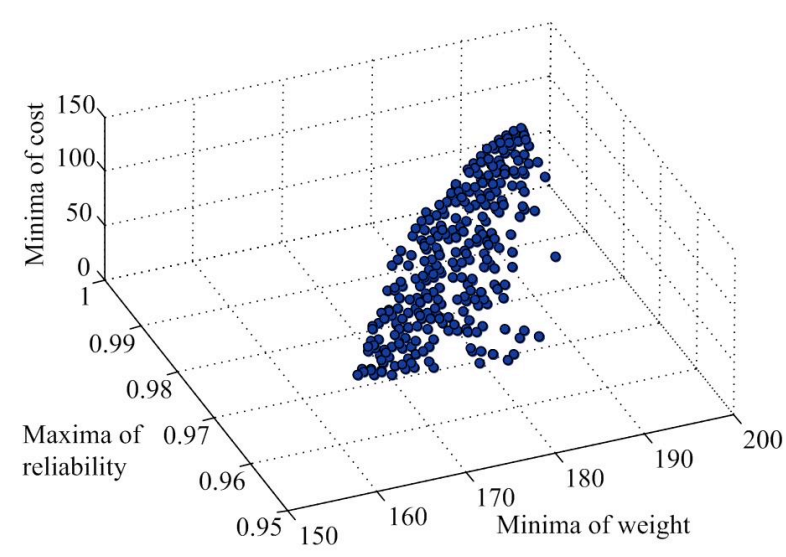

Fig. 10. Pareto front acquired by INSGA-II for RAP with three objectives

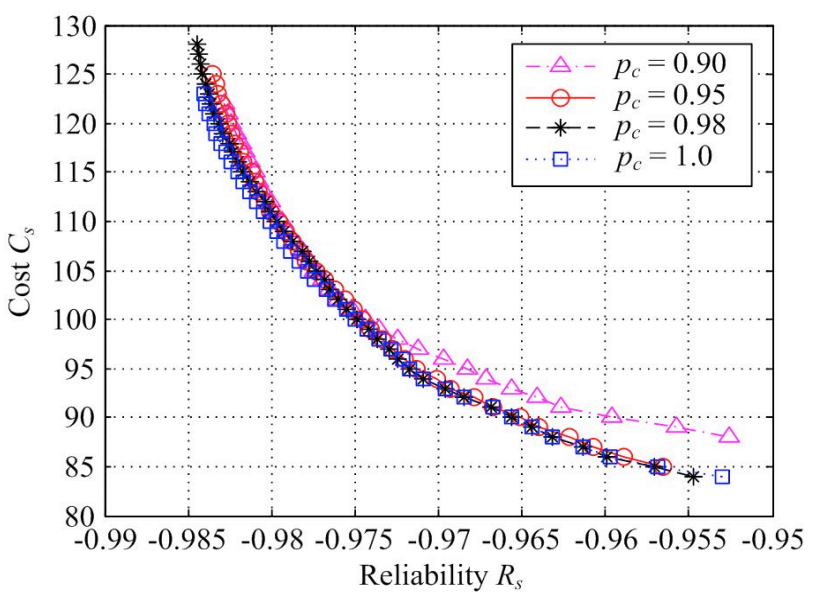

Fig. 11. Pareto fronts with different crossover probabilities $p_{c}$

The mutation probability was another important parameter that could affect the stability of INSGA-II. Fig. 12 shows the Pareto fronts for different mutation rates with the same two-objective optimization model (Formula (10)) and $p_{c}=0.98$. When $p_{m}=0.1$ and 0.3 , that is, $p_{m}$ with a large value, the convergence and diversity of the Pareto fronts were considerably worse than $p_{m}=0$. The convergence of $p_{m}=0$ was similar to the convergence in $p_{m}=1 / s$, but the uniform distribution of $p_{m}=0$ was slightly worse. Thus, the mutation probability should be set as a small value, e.g., $p_{m}=1 / \mathrm{s}$, to avoid over-vibration in the evolutionary process caused by large mutation rates.

\section{Engineering example of an agricultural IoT}

To illustrate the rationality and validity of the proposed INSGA-II further, an engineering example of an agricultural IoT is presented in this section. The system application of IoT greatly improved labor productivity and product quality in agricultural facilities with high inputs and outputs. However, in practice, system failures caused by power failure, sensor failure, network failure, control equipment damage, and other factors often lead to production accidents. A reasonable reliability redundancy technology must be adopted to ensure the system reliability and stability of IoT in agricultural facilities. Therefore, the proposed scheme is used to solve the reliability RAP of an agricultural IoT, 
which is established for greenhouse planting (Fig. 13). The system consists of sensors and actuators (e.g., air temperature and humidity sensor, light sensor, soil temperature and humidity sensor, $\mathrm{CO}_{2}$ sensor, ventilator, supplementary light lamp, and $\mathrm{CO}_{2}$ generator). Table 3 shows the detailed component (functional unit) information.

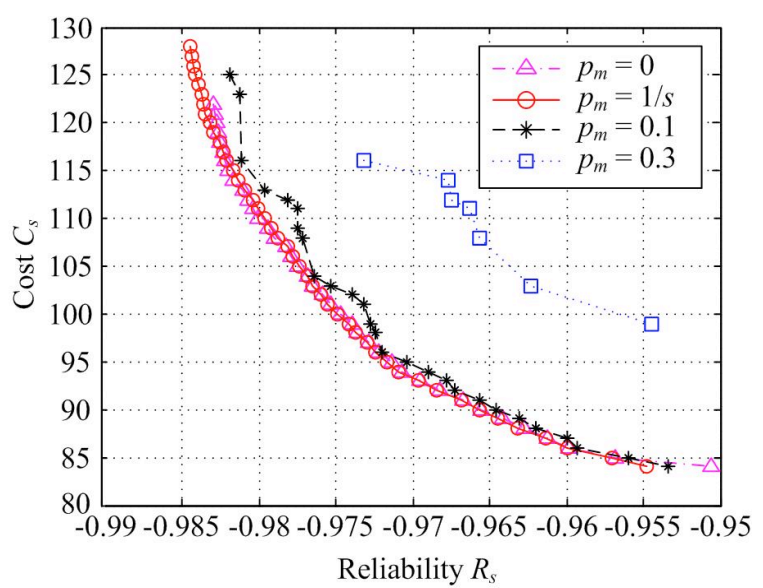

Fig. 12. Pareto fronts with different mutation probabilities $p_{m}$

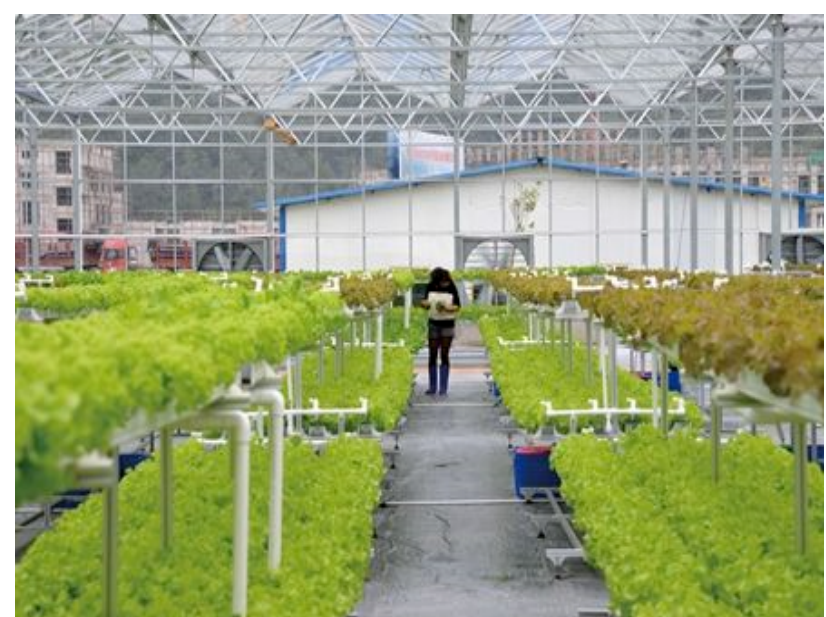

Fig. 13. Agricultural IoT for greenhouse planting

For the target IoT in agricultural facility, the maximum cost is $C_{\max }=140$, maximum weight is $W_{\max }=200, n=4$, and $n_{\min }=2$. Then, a two-objective model for the standard redundancy allocation test problem was constructed.

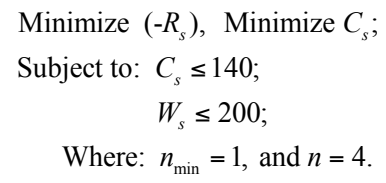

Table. 3. Numerical information for the reliability redundancy allocation model of the target agricultural IoT

\begin{tabular}{|c|c|c|c|c|c|c|c|c|c|c|c|c|c|}
\hline \multirow{3}{*}{ Functional unit } & \multirow{3}{*}{$\begin{array}{l}\text { Component } \\
\text { (i) }\end{array}$} & \multicolumn{12}{|c|}{ Design alternative $(j)$} \\
\hline & & \multicolumn{3}{|c|}{1} & \multicolumn{3}{|c|}{2} & \multicolumn{3}{|c|}{3} & \multicolumn{3}{|c|}{4} \\
\hline & & $R$ & $C$ & $W$ & $R$ & $C$ & $W$ & $R$ & $C$ & $W$ & $R$ & $C$ & $W$ \\
\hline $\begin{array}{l}\text { Air temperature and } \\
\text { humidity sensor }\end{array}$ & 1 & 0.92 & 3 & 2 & 0.91 & 2 & 4 & 0.94 & 4 & 8 & 0.88 & 1 & 1 \\
\hline Light sensor & 2 & 0.85 & 1 & 3 & 0.87 & 2 & 7 & 0.91 & 2 & 4 & 0.95 & 4 & 9 \\
\hline $\begin{array}{l}\text { Soil temperature and } \\
\text { humidity sensor }\end{array}$ & 3 & 0.77 & 1 & 5 & 0.92 & 3 & 3 & 0.85 & 2 & 2 & 0.96 & 4 & 7 \\
\hline $\mathrm{CO}_{2}$ sensor & 4 & 0.90 & 3 & 4 & 0.98 & 4 & 7 & 0.78 & 1 & 2 & 0.84 & 2 & 5 \\
\hline Ventilator & 5 & 0.95 & 4 & 2 & 0.86 & 2 & 4 & 0.90 & 2 & 4 & 0.92 & 3 & 2 \\
\hline Water machine & 6 & 0.85 & 2 & 7 & 0.79 & 1 & 9 & 0.92 & 3 & 5 & 0.96 & 4 & 3 \\
\hline Circulation machine & 7 & 0.81 & 1 & 4 & 0.87 & 2 & 8 & 0.94 & 4 & 3 & $*$ & * & $*$ \\
\hline Curtain rolling machine & 8 & 0.90 & 2 & 6 & 0.99 & 4 & 3 & 0.82 & 1 & 7 & 0.88 & 2 & 5 \\
\hline Sunscreen & 9 & 0.88 & 2 & 5 & 0.91 & 3 & 4 & 0.95 & 4 & 7 & 0.82 & 1 & 9 \\
\hline Supplementary lamp & 10 & 0.93 & 3 & 3 & 0.99 & 4 & 2 & 0.86 & 2 & 6 & 0.80 & 2 & 4 \\
\hline $\begin{array}{l}\text { Water pump with drip } \\
\text { irrigation solenoid valve }\end{array}$ & 11 & 0.99 & 4 & 5 & 0.92 & 3 & 3 & 0.85 & 1 & 8 & * & * & * \\
\hline $\begin{array}{l}\text { Water pump with micro- } \\
\text { injection solenoid valve }\end{array}$ & 12 & 0.86 & 2 & 3 & 0.79 & 1 & 5 & 0.96 & 4 & 4 & 0.81 & 2 & 7 \\
\hline $\mathrm{CO}_{2}$ generator & 13 & 0.79 & 1 & 9 & 0.87 & 2 & 4 & 0.90 & 4 & 5 & 0.97 & 5 & 3 \\
\hline
\end{tabular}

On the basis of the sensitivity analysis in Section 4.4, crossover probability $\mathrm{pc}$ and mutation probability $\mathrm{pm}$ are determined as 0.98 and $1 / s$, respectively. In the target model for agricultural IoT, $s=13$. The other relevant INSGA-II parameters for the two-objective RAP are set on the basis of Section 4.1.

The corresponding Pareto fronts acquired by INSGA-II for the target model for agricultural IoT can be obtained, as shown in Fig. 14. Table 4 presents the relevant results for the redundancy allocation optimization with two objectives using INSGA-II. Fig. 14 and Table 4 show that (a) the Pareto front with relatively good boundary and diversity characteristics for RAP with two objectives could be acquired by INSGA-II. (b) Neither the maxima of reliability only $R_{s}=0.9976$ nor the minima of cost only $C_{s}=71$ was superior to the baseline design in view of both objectives. (c) Compromised solutions $R_{s}=0.9833$ and $C_{s}=107$ acquired a higher reliability and lower cost than the baseline designs $R_{s}=0.9782$ and $C_{s}=128$ in which the optimization amounts were $0.52 \%$ and $16.4 \%$, respectively. Therefore, the proposed INSGA-II is effective and reasonable in solving the reliability redundancy problem to ensure the system reliability and stability of IoT in agricultural facility. 


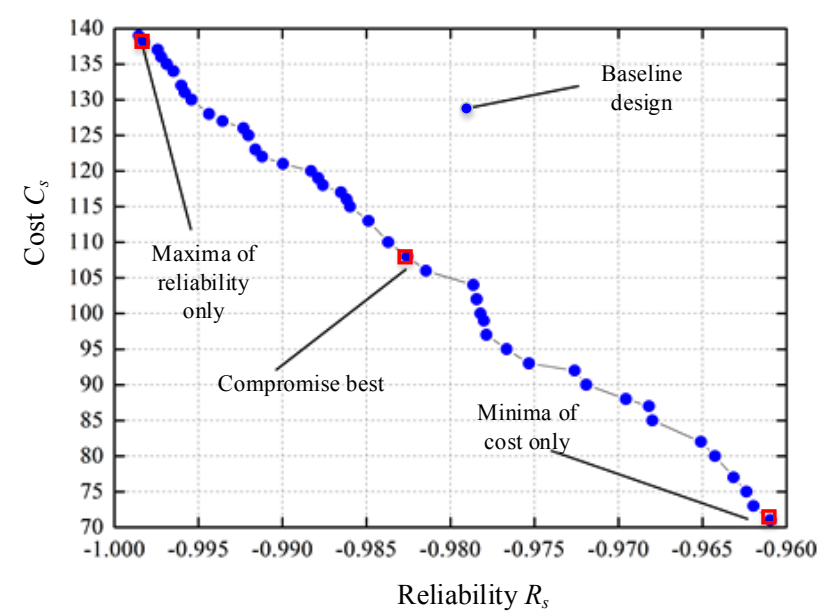

Fig. 14. Pareto fronts acquired by INSGA-II for RAP with two objectives of the IoT in agricultural facility.

Table 4. Results for redundancy allocation optimization with two objectives using INSGA-II of the IoT in agricultural facility

\begin{tabular}{c|c|c|c|c}
\hline Description & $\begin{array}{c}\text { Baseline } \\
\text { design }\end{array}$ & $\begin{array}{c}\text { Compromised } \\
\text { Best }\end{array}$ & $\begin{array}{c}\text { Minima } \\
\text { of } \\
\text { cost only }\end{array}$ & $\begin{array}{c}\text { Maxima of } \\
\text { reliability } \\
\text { only }\end{array}$ \\
\hline 1 & 4440 & 2220 & 2200 & 3300 \\
2 & 2330 & 1330 & 3100 & 3200 \\
3 & 4430 & 2230 & 3330 & 2200 \\
4 & 2440 & 3333 & 2300 & 1110 \\
5 & 3333 & 2200 & 4300 & 1130 \\
6 & 2220 & 1440 & 2430 & 3344 \\
7 & 2230 & 2110 & 3300 & 3330 \\
8 & 3044 & 4440 & 2222 & 1140 \\
9 & 4330 & 2233 & 1111 & 3300 \\
10 & 1112 & 2240 & 3320 & 4440 \\
11 & 3300 & 1300 & 2300 & 2200 \\
12 & 3344 & 2300 & 2444 & 4220 \\
13 & 1110 & 3200 & 3333 & 4330 \\
$R_{s}$ & 0.9782 & 0.9833 & 0.9622 & 0.9976 \\
$C_{s}$ & 128 & 107 & 71 & 139 \\
\hline$W_{s}$ & 185 & 113 & 189 & 194 \\
\hline
\end{tabular}

\section{Conclusions}

To explore the non-maintained bi-state series-parallel system with active redundancy and component mixing scheme and solve the RAP for series-parallel systems, a mathematical model for solving the redundant allocation of reliability was established. The RAP was encoded with the integer matrix. The related integer crossover, mutation, and adjusting operators were developed. Furthermore, an integer coding genetic algorithm was obtained to optimize the redundant allocation for series-parallel systems. The following conclusions could be drawn:

(1) A novel integer coding genetic scheme was proposed, and related integer crossover, mutation, and adjusting operators were developed and implemented for the first time in NSGA-II to solve the RAP with active redundancy and component mixing.

(2) The integer encoding redundancy allocation model was solved with traditional NSGA-II, in which constraint Pareto dominance was introduced to avoid the complexity and instability in penalty function approaches.

(3) A three-objective RAP model with maximized system reliability, minimized cost, and minimized weight was constructed. The proposed method was applied to a standard redundancy allocation test problem to compare three reliability objectives and select the most suitable one.

(4) INSGA-II had the best stability when the optimized value for the crossover probability was 0.98 and the mutation probability was set to a small value.

The integer matrix encoding, crossover, mutation, and adjusting operators provided a computational model for RAP. This model could be transplanted conveniently into other multi-objective genetic algorithms to solve the MORAP for serial-parallel systems easily; it had good suitability for genetic evolution platforms. However, the computational efficiency should be further improved. Therefore, further efficient evolutionary algorithms should be developed to solve multi-state RAP, thereby allowing the selection of redundancy strategies in future studies, and the redundant allocation of series-parallel systems should be further accurate.

\section{Acknowledgements}

This work was supported by the National Natural Science Foundation of China (Grant No. 51475148) and Young Backbone Teacher Training Program of Henan Colleges and Universities (Grant No. 2018GGJS173).

This is an Open Access article distributed under the terms of the Creative Commons Attribution License

\section{References}

1. Kuo, W., Wan, R., "Recent advances in optimal reliability allocation". IEEE Transactions on Systems Man and Cybernetics, 37(2), 2007, pp.143-156.

2. Azizmohammadi, R., Amiri, M., et al., "Solving a redundancy allocation problem by a hybrid multi-objective imperialist competitive algorithm". International Journal of Engineering, 26(9), 2013, pp.1025-2495.

3. Wang, W., Xiong, J., Xie, M., "Cold-standby redundancy allocation problem with degrading components". International Journal of General Systems, 44 (7-8), 2015, pp.876-888.

4. Ardakan, M. A., Hamadani, A. Z., "Reliability-redundancy allocation problem with cold-standby redundancy strategy". Simulation Modelling Practice \& Theory, 42(3), 2014, pp.107-118.

5. Teimouri, M., Zaretalab, A., et al., "An efficient memory-based electromagnetism-like mechanism for the redundancy allocation problem". Applied Soft Computing, 38(C), 2016, pp.423-436.
6. Coit, D. W., Smith, A. E., "Reliability optimization of series-parallel systems using a genetic algorithm". IEEE Transactions on Reliability, 45(2), 1996, pp.254-266.

7. Zhao, J. H., Liu, Z. H., Dao, M. T., "Reliability optimization using multi-objective ant colony system approaches". Reliability Engineering and System Safety, 92(1), 2007, pp.109-120.

8. Hsieh, T. J., Yeh, W. C., "Penalty guided bees search redundancy allocation problems with a mix of components in series-parallel systems". Computers \& Operations Research, 39(11), 2012, pp.2688-2704.

9. Chambari, A., Rahmati, A., et al., "A bi-objective model to optimize reliability and cost of system with a choice of redundancy strategies". Computers \& Industrial Engineering, 63(1), 2012, pp.109-119. 
10. Yeh, W. C., Luo, C. Y., et al., "Simplified swarm optimization with modular search for the general multi-level redundancy allocation problem in series-parallel systems". In: 2016 Congress on Evolutionary Computation, Vancouver, Canada: IEEE, 2016, pp.778-784.

11. Zio, E., Bazzo, R., "Level diagrams analysis of Pareto front for multi-objective system redundancy allocation". Reliability and System Engineering, 96(5), 2017, pp.569-580.

12. Liang, Y. C., \& Lo, M. H.: "Multi-objective redundancy allocation optimization using a variable neighborhood search algorithm". Journal of Heuristics, 16(3), 2010, pp.511-535.

13. Yeh, W. C., "Orthogonal simplified swarm optimization for the series-parallel redundancy allocation problem with a mix of components". Knowledge-Based Systems, 64(21), 2014, pp.1-12.

14. Feizollahi, M. J., Soltani, R., Feyzollahi, H., "The robust cold standby redundancy allocation in series-parallel systems with budgeted uncertainty". IEEE Transactions on Reliability, 64(2), 2015, pp.799-806.

15. Ardakan, M. A., Hamadani, A. Z., Alinaghian M., “Optimizing biobjective redundancy allocation problem with a mixed redundancy strategy". Retrieved from http://doi.org/10.1016/j.isatra, 2014-102/2019-2-28

16. Garg, H., Rani, M., Sharma, S. P., et al., "Bi-objective optimization of the reliability-redundancy allocation problem for series-parallel system". Journal of Manufacturing Systems, 33(3), 2014, pp.335347.

17. Yeh, W. C., Lin, J. S., "New parallel swarm algorithm for smart sensor systems redundancy allocation problems in the Internet of Things". Journal of Supercomputing, 74(9), 2018, pp.4358-4384.

18. Tavakkolimoghaddam, R., Safari, J., Sassani, F., "Reliability optimization of series-parallel systems with a choice of redundancy strategies using a genetic algorithm". Reliability Engineering \& System Safety, 93(4), 2017, pp.550-556.
19. Mousavi, S. M., Alikar, N., Niaki, S. T. A., "An improved fruit fly optimization algorithm to solve the homogeneous fuzzy seriesparallel redundancy allocation problem under discount strategies". Soft Computing, 20(6), 2016, pp.2281-2307.

20. Maciej, Laszczyk., Paweł, B., Myszkowski., "Improved selection in evolutionary multi-objective optimization of multi-skill resourceconstrained project scheduling problem". Information Sciences, 481(5), 2019, pp.412-431.

21. He, P., Zheng, Z. H., et al., "Optimization of mixed redundancy strategy with instant switching for series-parallel systems". Journal of Software, 28(2), 2017, pp.443-456.

22. Wang, J., Zhao, N., et al., "Application of particle swarm optimization and local search in structure optimization of seriesparallel system". Machinery and Electronics, 35(1) 2014, pp.18-21.

23. Mousavi, S. M., Alikar, N., Niaki, S. T. A., "An improved fruit fly optimization algorithm to solve the homogeneous fuzzy seriesparallel redundancy allocation problem under discount strategies". Soft Computing, 20(6), 2016, pp.2281-2307.

24. Jung, D., Choi, Y. H., Kim, J. H., "Multiobjective automatic parameter calibration of a hydrological model". Water, 9(3), 2017, pp. 1-23.

25. Fan, Z., Fang, Y., et al., "A comparative study of constrained multiobjective evolutionary algorithms on constrained multi-objective optimization problems". In: 2017 Conference Evolutionary Computation. San Sebastian, Spain: IEEE, 2017, pp. 209-216.

26. Ebrahimnejad, A., Verdegay, J. L., "Fuzzy sets-based methods and techniques for modern analytics". Cham: Springer International Publishing AG, Switzerland, 2018, pp.1-27.

27. Shukla, J., Das, B., Pant, V., "Consideration of small signal stability in multi-objective DS reconfiguration in the presence of distributed generation". Iet Generation Transmission \& Distribution, 11(1), 2017, pp.236-245. 